\title{
Minimal Supersymmetric Pati-Salam Theory: Determination of Physical Scales.
}

\author{
Alejandra Melfo ${ }^{(1,2)}$ and Goran Senjanović(1) \\ (1) International Centre for Theoretical Physics, 34100 Trieste, Italy and \\ (2) Centro de Astrofísica Teórica, Universidad de Los Andes, Mérida, Venezuela
}

\begin{abstract}
We systematically study the minimal supersymmetric Pati-Salam theory, paying special attention to the unification constraints. We find that the $S U(4)_{c}$ scale $M_{c}$ and the Left-Right scale $M_{R}$ lie in the range $10^{10} \mathrm{GeV}<M_{c}<10^{14} \mathrm{GeV}, 10^{3} \mathrm{GeV}<M_{R}<10^{10} \mathrm{GeV}$ (with single-step breaking at $10^{10} \mathrm{GeV}$ ), giving a potentially accessible scale of parity breaking. The theory includes the possibility of having doubly-charged supermultiplets at the supersymmetry breaking scale; color octet states with mass $\sim M_{R}^{2} / M_{c}$; magnetic monopoles of intermediate mass that do not conflict with cosmology, and a "clean" (type I) form for the see-saw mechanism of neutrino mass.
\end{abstract}

\section{INTRODUCTION}

The theories of Grand Unification owe their origin to the beautiful idea of quark-lepton symmetry put forward about thirty years ago by Pati and Salam [1]. The minimal and original realization is based on a $G_{P S}=$ $S U(2)_{L} \times S U(2)_{R} \times S U(4)_{c}$ symmetry, and has been studied thoroughly in the past (for recent work see for example 2, 3] and references therein). It is characterized by a number of interesting and important features

1. it incorporates Left-Right (LR) symmetry 1, 4, 5] (on top of the quark-lepton symmetry mentioned above), which leads naturally to the spontaneous breaking of parity and charge conjugation [5, 6].

2. incorporates a see-saw mechanism for small neutrino masses [7, 8, 9, 10]

3. predicts the existence of magnetic monopoles [11, 12,13

4. leads to rare processes such as $K_{L} \rightarrow \mu \bar{e}$ through the lepto-quark gauge bosons (with however a negligible rate for $M_{P S} \geq 10 \mathrm{GeV}$ ) [1]

5 . in the case of single-step breaking, predicts the scale of quark-lepton (and Left-Right) unification 14

6. allows naturally for $\Delta B=2$ process of $n-\bar{n}$ oscillations (with however a negligible rate unless there are light diquarks in the $\mathrm{TeV}$ mass region) 15, 16, 17, 18.

7. last but not least, it allows for implementation of the leptogenesis scenario, as suggested by the seesaw mechanism [19].

Since the scale of unification is large (see below), the Pati-Salam (PS) model can turn out to be indistinguishable from a less unifying theory, such as LR, or a more unifying one such as $\mathrm{SO}(10)$. The issue we want to address here is, what could be the smoking gun of a PatiSalam model?

We believe that this issue should be investigated in the context of the minimal PS theory. Of course, one wishes to appeal to supersymmetry as the usual protection mechanism for large hierarchies. Now, the supersymmetric standard model includes potentially catastrophic $d=4$ operators leading to proton decay. The supersymmetric PS model, as any model of the renormalizable see-saw mechanism, solves this problem since it leads to R-parity being an exact symmetry to all orders in perturbation theory 20]. As a bonus, the LSP is stable and becomes a natural candidate for the dark matter of the universe.

We will therefore systematically study the minimal supersymmetric Pati-Salam theory, paying special attention to possible low energy predictions. We allow for two-step breaking

$$
\begin{array}{r}
S U(2)_{L} \times S U(2)_{R} \times S U(4)_{c} \\
\longrightarrow S U(2)_{L} \times S U(2)_{R} \times U(1)_{B-L} S U(3)_{c} \\
\longrightarrow S U(2)_{L} \times U(1)_{Y} \times S U(3)_{c}
\end{array}
$$

Our findings are as follows. If one ignores nonrenormalizable terms, the $M_{c}$ scale is in the range

$$
10^{10} \mathrm{GeV}<M_{c}<10^{14} \mathrm{GeV}
$$

while at the same time

$$
10^{10} \mathrm{GeV}>M_{R}>10^{3} \mathrm{GeV}
$$

In other words, lower $M_{R}$ implies larger $M_{c}$. We find it remarkable that unification allows $M_{R}$ to be close to the experimental limit, and thus potentially observable.

Of course, the lower $M_{R}$, the more fine-tuning needed in order to have neutrinos light. But still, strictly speaking, Dirac neutrino mass can be arbitrary, and thus neutrinos could be light. At the same time $M_{c}$ is pushed to its upper limit $\sim 10^{14} \mathrm{GeV}$ which leads to the well-known monopole problem of theories with symmetry breaking scales above $10^{11} \mathrm{GeV}$. One can invoke inflation (or some other mechanism, 21, 22, 23] ) to solve the problem, but this simply renders the $S U(4)_{c}$ symmetry invisible.

Another extreme is the single-step breaking with $M_{R} \sim M_{c} \simeq 10^{10} \mathrm{GeV}$. In this case monopoles have a mass $m_{M} \simeq 10^{11} \mathrm{GeV}$, and they are perfectly compatible with all the experimental, astrophysical and cosmological data. At the same time one has the usual leptogenesis scenario 24] and naturally small neutrino masses; 
and furthermore $S U(4)_{c}$ could be observable through the possible discovery of magnetic monopoles.

Another interesting consequence of this theory is a "clean" (type I) see-saw mechanism, as we discuss later.

Most important, this theory includes the possibility of existence of light doubly charged supermultiplets (as noticed in the past [25, 26]) with masses in the $\mathrm{TeV}$ region, and a potentially light (for $M_{R} \ll M_{c}$ ) color octet supermultiplet with mass $\sim M_{R}^{2} / M_{c}$, which could also be as low as $T e V$. Relatively light color octets are known to be present in other theories. Even in the minimal supersymmetric $S U(5)$ they could lie much below the GUT scale [27].

Non-renormalizable effects suppressed by $M_{P l}$ become important if $M_{R}^{2}>M_{W} M_{P l}$. In this case, the single-step breaking at $M_{R} \sim M_{c} \simeq 10^{10} \mathrm{GeV}$ turns out to be the only possibility, and the doubly charged supermultiplets become potentially observable with masses in the $\mathrm{TeV}$ range. However, the color octet becomes heavy, making the theory indistinguishable from the LR model.

A more likely possibility is the existence of nonrenormalizable terms cut-off by a much lower scale than $M_{P l}$. Namely, the theory is not asymptotically free above $M_{c}$, and the gauge coupling becomes strong at $M_{F} \simeq$ $10 M_{c}$. Including $1 / M_{F}$ terms (with $M_{R}^{2}>M_{W} M_{F}$ ) leads to $M_{R} \geq 10^{7} \mathrm{GeV}, M_{c} \geq 10^{12} \mathrm{GeV}$, and the color octet and doubly-charged multiplets have the same mass, $\sim M_{R}^{2} / M_{c}$. Again, these particles could be observable and reveal four colour unification. In what follows we discuss these findings at length.

\section{THE MODEL: MINIMAL PS THEORY WITH TWO-STEP BREAKING}

It is easy to see that (11) can be achieved with the following minimal set of Higgs-like supermultiplets: (the numbers in parenthesis indicate the $G_{P S}$ representations)

$$
\begin{gathered}
A(1,1,15) \\
\Sigma(3,1,10), \bar{\Sigma}(3,1, \overline{10}), \Sigma_{c}(1,3, \overline{10}), \bar{\Sigma}_{c}(1,3,10)
\end{gathered}
$$

The matter supermultiplets are

$$
\psi(2,1,4), \psi_{c}(1,2, \overline{4})
$$

and the minimal light Higgs multiplet is

$$
\phi(2,2,1)
$$

The most general superpotential for the fields (4) is

$W=m \operatorname{Tr} A^{2}+\operatorname{MTr}\left(\Sigma \bar{\Sigma}+\Sigma_{c} \bar{\Sigma}_{c}\right)+\operatorname{Tr}\left(\Sigma A \bar{\Sigma}-\Sigma_{c} A \bar{\Sigma}_{c}\right)$

where we assume the following transformation properties under Parity

$$
\Sigma \rightarrow \Sigma_{c}, \quad \bar{\Sigma} \rightarrow \bar{\Sigma}_{c}, \quad A \rightarrow-A
$$

We choose $A$ to be a parity-odd field in order to avoid flat directions connecting Left- and Right-breaking minima.
Notice that under $S U(3)_{c}$, the symmetric representation 10 of $S U(4)_{c}$ is decomposed as $10=6+3+$ 1. Clearly, only the singlets of $S U(3)_{c}$ in the $\Sigma$ fields, which we shall denote as $\Delta, \bar{\Delta}, \Delta_{c}$ and $\bar{\Delta}_{c}$, can take nonvanishing vevs.

This allows for the supersymmetry-preserving symmetry breaking pattern

$$
\begin{gathered}
<A>=M_{c} \operatorname{diag}(1,1,1,-3) \\
<\Delta>=<\bar{\Delta}>=0 \\
<\Delta_{c}>=M_{R}\left(\begin{array}{ll}
0 & 1 \\
0 & 0
\end{array}\right), \quad<\bar{\Delta}_{c}>=M_{R}\left(\begin{array}{ll}
0 & 0 \\
1 & 0
\end{array}\right)(11)
\end{gathered}
$$

(where the matrix in (9) is in $S U(4)$ space and those of (11) are in $S U(2)_{R}$ space), with

$$
M_{c} \simeq M, \quad M_{R} \simeq \sqrt{M m}
$$

The mass spectrum is easy to compute

\begin{tabular}{l|l}
$M_{c}$ & $\begin{array}{l}\text { all states in } \Sigma, \bar{\Sigma} ; \\
\text { the states in } A \text { except for an octet of color } \\
\text { the states in } \Sigma_{c}, \bar{\Sigma}_{c} \text { except for } \Delta_{c} \text { and } \bar{\Delta}_{c}\end{array}$ \\
$M_{R}$ & $\begin{array}{l}\text { the fields } \Delta_{c}(1,3,1) \text { and } \bar{\Delta}_{c}(1,3,1) \text { of } \Sigma_{c}, \bar{\Sigma}_{c}, \\
\text { except for the components } \delta_{c}^{++}, \bar{\delta}_{c}^{++} \\
\text {and a combination of the singlet components }\end{array}$ \\
$M_{R}^{2} / M_{c}$ & $\begin{array}{l}\text { color octet in A } \\
\Lambda_{S U S Y}\end{array}$ \\
$\begin{array}{l}\text { supermultiplets } \delta_{c}^{++}, \bar{\delta}_{c}^{++} \\
\text {and a combination of the singlets in } \Delta_{c} \text { and } \bar{\Delta}_{c} \\
\text { (and all the MSSM superpartners) }\end{array}$
\end{tabular}

Some comments are in order. The color octet mass is clear, $m \sim M_{R}^{2} / M_{c}$, but the situation with $\delta_{c}^{++}, \bar{\delta}_{c}^{++}$ is more subtle, and although it has been discussed before [26] it is worth repeating here. The fields $\Delta_{c}(1,3,1)$ and $\bar{\Delta}_{c}(1,3,1)$ responsible for the scale $M_{R}$ are coupled to the $(1,1,1)$ field in $A$ and so appear in the superpotential only through terms with $\operatorname{Tr} \Delta_{c} \bar{\Delta}_{c}$. This implies a larger, accidental $S U(3)$ symmetry broken down to $S U(2)$, hence five Nambu-Goldstone bosons. But the gauge symmetry $S U(2)_{R} \times U(1)_{B-L}$ is broken down to $U(1)_{Y}$, so that three of them are eaten, leaving us with massless states $\delta_{c}^{++}, \bar{\delta}_{c}^{++}$. Of course, they become massive when supersymmetry gets broken, and so they have masses of order $\mathrm{TeV}$. This is the most interesting prediction of SUSY LR (PS) theories.

However, there is a problem associated with this. Namely, the charge-preserving vacuum lies on a flat direction 28]

$$
<\Delta_{c}>=M_{R}\left(\begin{array}{cc}
0 & \cos \theta \\
\sin \theta & 0
\end{array}\right) \quad<\bar{\Delta}_{c}>=M_{R}\left(\begin{array}{cc}
0 & \sin \theta \\
\cos \theta & 0
\end{array}\right)
$$


(in other words, the potential is $\theta$-independent, and charge conservation requires $\theta=0$ or $\theta=\pi / 2)$. If the soft breaking terms break also $S U(2)_{R}$, then, of course, one may eliminate the charge breaking vacua. If not, one would have to appeal to higher dimensional operators (which in general break the accidental $S U(3)$ symmetry). We discuss this possibility in Section IV.

On top of the above states are the usual quarks and leptons in

$$
\psi(2,1,4) ; \quad \psi_{c}(1,2, \overline{4})
$$

and the two MSSM Higgs doublet superfields belonging to a combination of the $(2,2,1)$ and the $(2,2,15)$ fields (see below). The fermions $\psi$ and $\psi_{c}$ provide a complete representation (we assume no new matter states) and can be used to normalize the $U(1)$ generators, such as $Y$ or $B-L$.

\section{UNIFICATION CONSTRAINTS}

From the charge formula in LR symmetric theories, we have $Y / 2=I_{3 R}+(B-L) / 2$. Using the fact that $(B-L) / 2$ is a generator of $S U(4)_{c}$, we can properly normalize the generators. It is then easy to see that the combination

$$
\Delta \alpha^{-1} \equiv \alpha_{Y}^{-1}-\alpha_{L}^{-1}-\frac{2}{3} \alpha_{c}^{-1}
$$

(where $\alpha_{Y}, \alpha_{L}$ and $\alpha_{c}$ are the $U(1)_{Y}, S U(2)_{L}$ and $S U(3)_{c}$ couplings respectively) is a function of only $M_{R}$ and $M_{c}$.

In what follows, we perform a one-loop analysis, and in order to estimate $M_{c}$ and $M_{R}$ we can safely ignore the difference between $M_{W}$ and $\Lambda_{S U S Y}$ noting that it becomes relevant at the two-loop level. It is then easy to show that

a) for $M_{R}^{2} \geq M_{W} M_{c}$,

$$
2 \pi \Delta \alpha^{-1}=12 \ln \frac{M_{c}}{M_{W}}+8 \ln \frac{M_{R}}{M_{W}},
$$

and

b) for $M_{R}^{2} \leq M_{W} M_{c}$,

$$
2 \pi \Delta \alpha^{-1}=14 \ln \frac{M_{c}}{M_{W}}+4 \ln \frac{M_{R}}{M_{W}} .
$$

In the MSSM, one would get

$$
2 \pi \Delta \alpha^{-1}=12 \ln \frac{M_{U}}{M_{W}}
$$

where $M_{U}$ is the unification scale, $M_{U} \simeq 10^{16} \mathrm{GeV}$.

It is easy to see that in this approximation

$$
10^{10} \mathrm{GeV} \leq M_{c} \leq 10^{14} \mathrm{GeV}
$$

while at the same time

$$
10^{10} \mathrm{GeV} \geq M_{R} \geq 10^{3} \mathrm{GeV}
$$

As it is clear from (16) and (17), lower $M_{R}$ implies bigger $M_{c}$. It is remarkable that unification constraints allow for the parity breaking scale to be experimentally detectable. Of course since the see-saw mechanism requires $m_{\nu} \propto M_{R}^{-1}$, small neutrino masses prefer larger $M_{R}$.

A comment is noteworthy here. This is true if we assume that $m_{D}$ (the Dirac neutrino mass) is of the order of the charged lepton or quark masses. In the minimal theory one is tempted to use only a $(2,2,1)$ Higgs to break $S U(2)_{L} \times U(1)_{Y}$. Then one has

$$
m_{D}=m_{u}, \quad m_{\ell}=m_{d}
$$

for the down quarks $(d)$, charged leptons $(\ell)$, up quarks $(u)$ and neutrino Dirac $(D)$ masses. But $m_{\ell}=m_{d}$ (at $M_{c}$ ) fails badly for the first two generations and so (21) cannot really be trusted. One can add a $(2,2,15)$ in order to correct (21), in which case the mass spectrum looks like

$$
\begin{aligned}
m_{u} & =y^{1} v_{u}^{1}+y^{15} v_{u}^{15} \\
m_{D} & =y^{1} v_{u}^{1}-3 y^{15} v_{u}^{15} \\
m_{d} & =y^{1} v_{d}^{1}+y^{15} v_{d}^{15} \\
m_{\ell} & =y^{1} v_{d}^{1}-3 y^{15} v_{d}^{15}
\end{aligned}
$$

Clearly, the theory can be made realistic without any change in the unification predictions since only one bidoublet remains light after the usual fine-tuning. The price is arbitrariness at $m_{D}$ and the inability to predict neutrino masses. Low $M_{R}$ is then phenomenologically allowed and in accord with $S U(4)_{c}$ unification.

A positive note. As is well-known, in LR theories the see-saw mechanism is not type I, i.e. it contains an additional piece $\propto<\Delta>\simeq M_{W}^{2} / M_{R}[29$, 30]. What happens is the following. The symmetry allows for a coupling in the potential

$$
\Delta V=\lambda \Delta \phi^{2} \Delta^{c}+M^{2} \Delta^{2}
$$

which gives a small vev to $\Delta$

$$
<\Delta>=\lambda \frac{<\phi>^{2} M_{R}}{M^{2}} \simeq \lambda \frac{M_{W}^{2}}{M_{R}}
$$

Now, in supersymmetry this does not happen, at least at the renormalizable level. We can have higherdimensional terms in the superpotential

$$
\Delta W=\frac{1}{M_{P l}} \Delta \phi^{2} \Delta^{c}
$$

which imply a tiny, negligible vev $<\Delta>\propto M_{W}^{2} / M_{P l}$.

One could also generate such terms if there are interactions

$$
W=\phi^{2} S+\Delta \Delta^{c} S+M S^{2}
$$

where $S=(3,3,1)$ under $G_{P S}$. Integrating out $S$ would then give (25) with $M_{P l} \rightarrow M$. 
Another possibility would be

$$
W=\phi \Delta X+\phi \Delta^{c} \bar{X}+M X \bar{X}
$$

where $X=2,2, \overline{1} 0)$ and $\bar{X}=2,2,10)$ under $G_{P S}$. The absence of the $S, X, \bar{X}$ fields guarantees a type I see-saw at the supersymmetric level.

Now, once supersymmetry is broken, one can generate a nonvanishing but negligible vev for $\Delta[20]$ :

$$
<\Delta>\simeq\left(\frac{m_{3 / 2}}{M_{c}}\right)^{2} \frac{m_{D}^{2}}{M_{R}}
$$

which contributes by a tiny factor $\left(m_{3 / 2} / M_{c}\right)^{2} \leq 10^{-14}$ to the usual see-saw mass term $m_{\nu} \simeq m_{D}^{2} / m_{\nu_{R}}$.

In short, the see-saw mechanism takes its canonical form in the minimal model. Of course, since we do not know $m_{D}$ and the right-handed neutrino masses we cannot predict neutrino masses precisely, but the type I form serves for the leptogenesis scenario. Namely, in this case it is the Dirac Yukawa couplings which are responsible for bringing the right-handed neutrinos into equilibrium and also for their decay. Thus one can set constraints on $m_{D}$ and right-handed neutrino masses.

\section{EFFECTS OF NON-RENORMALIZABLE TERMS}

The crucial ingredient in obtaining (19) and (20) is the existence of doubly charged supermultiplets at the scale $\Lambda_{S U S Y} \simeq T e V$. We have not considered higher dimensional operators of the form $\left(\Delta^{c} \bar{\Delta}^{c}\right)^{2} / M_{P l}$, which are likely to be present and would cure the problem of potential breaking of the electromagnetic charge [31], even if the SUSY breaking terms preserve $S U(2)_{R}$. These terms would also give a mass to the doubly charged states, relevant for $M_{R}^{2} \geq M_{W} M_{P l}$. The unification constraint is now

$$
2 \pi \Delta \alpha^{-1}=12 \ln \frac{M_{c}}{M_{W}}-8 \ln \frac{M_{r}}{M_{W}}+8 \ln \frac{M_{P l}}{M_{W}}
$$

The only consistent solution is the single-step breaking

$$
M_{R} \simeq M_{c} \simeq 10{ }^{10} \mathrm{GeV}
$$

which guarantees the lightness of the doubly-charged supermultiplets. Since the color octets however become heavy, it is hard to distinguish this theory from the LR models. The only hope would be to find the relatively light magnetic monopoles, but it is hard to imagine an effective production mechanism in the context of supersymmetry.

Now, could there be another source of these masses? In principle, yes. Namely, the large representations we need to achieve the symmetry breaking imply the loss of asymptotic freedom above $M_{c}$. In fact, the gauge coupling becomes strong at the scale $M_{F} \simeq 10 M_{c}$. Thus, strictly speaking we could imagine operators of the type
$\left(\Delta^{c} \bar{\Delta}^{c}\right)^{2} / M_{F}$ which give a mass $M_{R}^{2} / 10 M_{c}$ to the doublycharged states. With the color octets and doubly charged particles having basically the same mass, the unification constraint turns out to be

$$
2 \pi \Delta \alpha^{-1}=20 \ln \frac{M_{c}}{M_{W}}-8 \ln \frac{M_{r}}{M_{W}}
$$

Keeping in mind that these effects are important for $M_{R}^{2} \geq M_{c} M_{W}$, one gets

$10^{12} \mathrm{GeV} \leq M_{c} \leq 10^{16} \mathrm{GeV} ; \quad 10^{7} \mathrm{GeV} \leq M_{R} \leq 10^{16} \mathrm{GeV}$

In other words, for $M_{R} \leq 10^{7} \mathrm{GeV}$, the colour octets and doubly charged states lie at the $\mathrm{TeV}$ scale, since the non-renormalizable terms play no role whatsoever. For $M_{R}$ bigger than $10^{7} \mathrm{GeV}$, it becomes harder to find these states, but they still remain comparable in mass. This could be the smoking gun of supersymmetric Pati-Salam theory.

\section{SUMMARY AND OUTLOOK}

The minimal renormalizable supersymmetric PatiSalam theory offers the exciting possibility of low scale parity restoration, even as low as $T e V$, thus making it accessible to experiment. The important, crucial prediction of the theory is the existence of doubly charged and color octet supermultiplets with an almost degenerate mass. The discovery of these states would be a signal of four colour unification.

As discussed in the paper, for $10^{3} \mathrm{GeV} \leq M_{R} \leq$ $10^{7} \mathrm{GeV}\left(10^{12} \mathrm{GeV} \leq M_{c} \leq 10^{14} \mathrm{GeV}\right)$, these particles could be discovered by $\mathrm{LHC}$ at the $T e V$ scale. In all honesty, such a low $M_{R}$ requires some fine-tuning in order to achieve a small neutrino mass. For larger $M_{R}$ these particles become heavier and less accessible to experiment, however non-renormalizable effects suppressed by a fundamental scale around $10 M_{c}$ still guarantee that they have comparable masses.

Breaking of PS symmetry at $M_{C}$ implies the existence of $U(1)_{B-L}$ monopoles. with mass $m_{M} \simeq 10 M_{c}$. If produced in a phase transition via the Kibble mechanism, the requirement that their density be less than the critical density then implies $M_{c} \leq 10^{12} \mathrm{GeV}$. In other words, if non-renormalizable terms cut off by $M_{F} \sim 10 M_{c}$ are present, we have the usual GUT (superheavy) monopole and one can invoke inflation or some other mechanism in order to get rid of them. Unfortunately, in this case the number of monopoles, if not zero, is not predictable at all. The single-step breaking at $M_{c} \sim M_{R} \sim 10^{10} \mathrm{GeV}$, on the other hand, offers the interesting possibility of potentially detectable intermediate mass monopoles. This however is a delicate point, since as is usual in supersymmetric theories, high-temperature effects would lead to a false vacuum problem. Namely, the phase transition may not occur at all, rending the theory unrealistic (for recent work and references see 32]). Whether if this can 
be avoided and an estimate of the monopole density can be made is beyond the scope of this paper.

\section{Acknowledgments}

We thank Borut Bajc for discussions. The work of A.M. was partially supported by CDCHT-ULA (Project
C-1073-01-05-A). The work of G.S. is partially supported by EEC (TMR contracts ERBFMRX-CT960090 and HPRN-CT-2000-00152). A.M. wishes to thank ICTP for hospitality during the course of this work.
[1] J. Pati and A. Salam, Phys. Rev. D10, 275 (1974).

[2] K. S. Babu and R. N. Mohapatra, Phys. Lett. B518, 269 (2001), hep-ph/0108089.

[3] R. Jeannerot, S. Khalil, G. Lazarides, and Q. Shafi, JHEP 10, 012 (2000), hep-ph/0002151.

[4] R. Mohapatra and J. Pati, Phys. Rev. D11, 2558 (1975).

[5] G. Senjanović and R. Mohapatra, Phys. Rev. D12, 1502 (1975).

[6] G. Senjanović, Nucl. Phys. B153, 334 (1979).

[7] M. Gell-Mann, P. Ramond, and R. Slansky, in Supergravity, edited by P. van Niewenhuizen and D. Freedman (North Holland, Amsterdam, 1979).

[8] T. Yanagida, in Workshop on Unified Theory and Baryon number in the Universe, edited by O. Sawada and A. Sugamoto (KEK, Japan, 1979).

[9] R. N. Mohapatra and G. Senjanović, Phys. Rev. Lett. 44, 912 (1980).

[10] S. Glashow, in Cargese 1979, Proceedings, Quarks and Leptons (1979), p. 687.

[11] A. M. Polyakov, JETP Letter 20, 194 (1974).

[12] G. 't Hooft, Nucl. Phys. B79, 276 (1974).

[13] A. Sen, Phys. Lett. B153, 55 (1985).

[14] J. C. Pati, A. Salam, and U. Sarkar, Phys. Lett. B133, 330 (1983).

[15] V. Kuzmin, JETP Lett 12, 228 (1970).

[16] S. Glashow, in Proceedings of Neutrino 'r9, edited by A. Haafut and C. Jarlskog (1979), p. 518.

[17] R. N. Mohapatra and R. E. Marshak, Phys. Rev. Lett.
44, 1316 (1980).

[18] Z. Chacko and R. N. Mohapatra, Phys. Rev. D59, 055004 (1999), hep-ph/9802388.

[19] M. Fukugita and T. Yanagida, Phys. Lett. B174, 45 (1986).

[20] C. S. Aulakh, A. Melfo, A. Rasin, and G. Senjanovic, Phys. Lett. B459, 557 (1999), hep-ph/9902409.

[21] P. Langacker and S.-Y. Pi, Phys. Rev. Lett. 45, 1 (1980).

[22] G. Dvali, A. Melfo, and G. Senjanović, Phys. Rev. Lett. 75, 4559 (1995), hep-ph/9507230.

[23] G. R. Dvali, H. Liu, and T. Vachaspati, Phys. Rev. Lett. 80, 2281 (1998), hep-ph/9710301.

[24] L. Boubekeur (2002), hep-ph/0208003.

[25] C. S. Aulakh, A. Melfo, and G. Senjanović (1997), hepph/9707256.

[26] Z. Chacko and R. N. Mohapatra (1997), hep-ph/9712359.

[27] C. Bachas, C. Fabre, and T. Yanagida, Phys. Lett. B370, 49 (1996), hep-th/9510094.

[28] R. Kuchimanchi and R. N. Mohapatra, Phys. Rev. D48, 4352 (1993), hep-ph/9306290.

[29] M. Magg and C. Wetterich, Phys. Lett. B94, 61 (1980).

[30] R. N. Mohapatra and G. Senjanović, Phys. Rev. D23, 165 (1981).

[31] R. N. Mohapatra and A. Rasin, Phys. Rev. Lett. 76, 3490 (1996), hep-ph/9511391.

[32] B. Bajc, I. Gogoladze, R. Guevara, and G. Senjanovic, Phys. Lett. B525, 189 (2002), hep-ph/0108196. 